\title{
Approach to novel functional foods for stress control 5. Antioxidant activity profiles of antidepressant herbs and their active components
}

\author{
Mutsuko Shirai, Yoshichika Kawai, Rintaro Yamanishi, and Junji Terao \\ Department of Food Science, Institute of Health Biosciences, The University of Tokushima Graduate \\ School, Tokushima, Japan
}

\begin{abstract}
Oxidative stress is frequently mentioned in relation to the neurodegenerative diseases. This study examined the effect of three herb extracts, Hypericum perforatum, Ginkgo biloba $L$. and Apocynum venetum $L$., and their components on lipid hydroperoxide-induced oxidative stress in PC-12cells. Among them, the extract of Apocynum venetum and its components showed the remarkable inhibitory effect, indicating that this herb extract serves as a protective agent against lipid peroxidation-related oxidative stress in CNS. Oxidative stress may be associated with the progress of depression, as this extract has been proposed to be an effective antidepresant herb. J. Med. Invest. 52 Suppl. : 249-251, November, 2005
\end{abstract}

Keywords : herb extract, antidepressant activity, antioxidant, $P C-12$, oxidative stress

\section{INTRODUCTION}

In recent years, herbs and herb-derived products have attracted much attention in relation to the prevention of depressive disorders. In particular, extract of Hypericum perforatum (St. John's Wort : $\mathrm{SJW}$ ) and that of Ginkgo biloba L. (GB) are frequently mentioned as antidepressant drugs and used at therapeutic level (1). Apocynum venetum $L(\mathrm{AV})$ is also implied to possess an anti-depressant activity by the result of forced swimming test (FST) of rats (2) , which is commonly used for the screening of antidepressant drugs. Their active components are not clarified yet, although phenolic compounds including hypericin and hyperforin (SJW), quercetin and kaempferol (GB) , and hyperoside and isoquercitrin (AV) are regarded as possible candidates for the

Received for publication September 9, 2005 ; accepted September $16,2005$.

Address correspondence and reprint requests to Junji Terao, Ph.D., Department of Food Science, Institute of Health Biosciences, The University of Tokushima Graduate School, Kuramoto-cho, Tokushima 770-8503, Japan, and Fax : +81-88633-7089. activity. On the other hand, central nervous system disorders including dementia and Alzheimer's disease, and decline of cognitive function are known to be associated with oxidative stress occurring in nerve cells. This prompted us to evaluate the antioxidant activity of the three herb extracts and their active components in nerve cells, because oxidative stress may be involved in the cellular mechanism of depressive disorders. Thus, we examined the efficacy of these herb extracts and active components to inhibit lipid hydroperoxide-induced production of reactive oxygen species (ROS) in PC-12 cultured cells.

\section{MATERIALS AND METHODS}

The extracts of SJW, GB and AV were kindly supplied from Tokiwa Phytochemical Co., Ltd (Chiba, Japan). Free radical scavenging activity of the herb extract was assayed using a stable free radical, 1, 1-diphenyl-2-picryllhydrazyl (DPPH) according to the method of Blois (3) and expressed as $\mu \mathrm{mol}$ cysteine equivalent per $\mathrm{mg}$ extract. PC-12 cells 
were differentiated by treating with nerve growth factor-7 S (NGF) for 7 days with medium replenishment every 2-3 days. The generation of intracellular ROS was evaluated by the fluorescence method using 2'7'-dichlorofluorescein (DCFH) (4). The fluorescence was monitored $1 \mathrm{hr}$ after the addition of 13hydroperoxy octadecadienoic acid (13-HPODE) (final conc. of $10 \mu \mathrm{M})$. In the case of the concurrent addition experiment, the cells were incubated with13-HPODE and the herb extract at $37^{\circ} \mathrm{C}$.

\section{RESULTS AND DISCUSSION}

Three herb extracts showed substantial DPPH radical scavenging activity (Table 1 ). The effectiveness of each extract was increased in the order of $\mathrm{GB}<\mathrm{SJW}<\mathrm{AV}$. Fig. 1 shows the result of concurrent addition experiment, in which $\mathrm{AV}$ exhibited the highest inhibitory effect on ROS production among the three extracts. Both GB and SJW showed the inhibition at higher concentration. In the pretreatment experiment in which the extract was exposed to PC-12 cells and washed out (5), only AV showed the remarkable inhibition in 13-HPODE-induced intracellular ROS production in the range of 1 to $100 \mu \mathrm{g} / \mathrm{ml}$ cell suspension (Fig. 2).

Hyperoside and isoquercitrin, which are involved in the active components for antidepressant activity of $\mathrm{AV}$ extract, are glycoside derivatives of antioxidative quercetin. We found that both compounds inhibited 13-HPODE-induced ROS production in PC-12 cells significantly (data are not shown here). It can be therefore concluded that $\mathrm{AV}$ extract is rich in powerful antioxidants. It is also suggested that AV extracts can serve as protective reagents against lipid peroxidationmediated oxidative stress in nerve cells, if they are able to reach to brain tissue across the blood-brain barrier (6) . On the assumption that oxidative stress is tightly associated with the progress of depression and mental stress-related diseases, our results imply that dietary intake of a certain plant food rich in phenolic antioxidants is helpful in the prevention of such diseases. We are now investigating the

Table 1. DPPH radical scavenging activity of herb extracts

\begin{tabular}{lc}
\hline \multicolumn{1}{c}{ extract } & $\mu \mathrm{mol}$ Cysteine $(\mathrm{eq} / \mathrm{mg})$ \\
\hline Apocynum venetum. (AV) & $8.54 \pm 0.04$ \\
Hypericum perforatum (SJW) & $3.22 \pm 0.03$ \\
Ginko biloba L (GB) & $3.04 \pm 0.01$ \\
Green tea extract & $2.99 \pm 0.01$ \\
Soybean flour & no activity \\
\hline
\end{tabular}

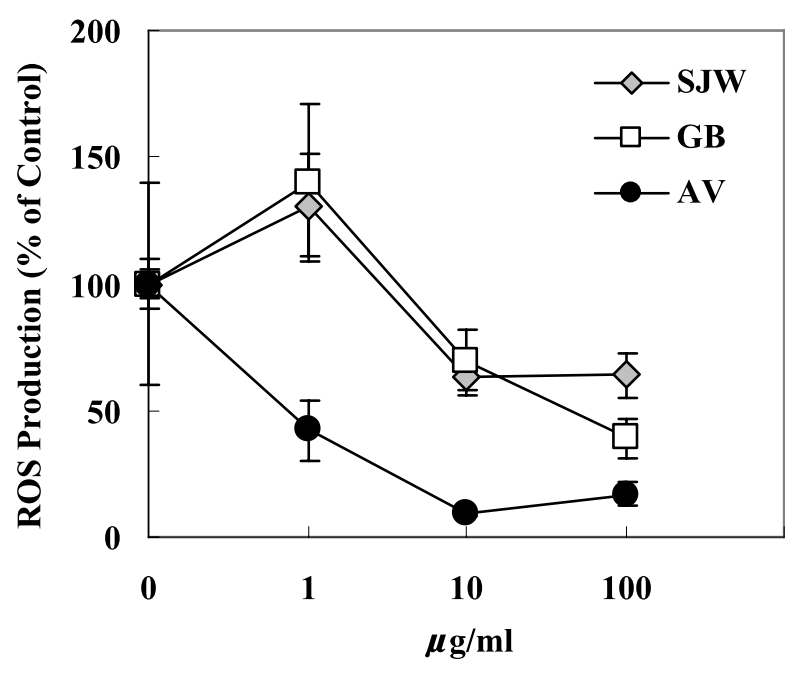

Fig.1. Effect of concurrent addition of herb extract on 13HPODE-induced intracellular production of ROS in PC-12 cells.

\section{$\triangle \mathrm{SJW} \quad \square \mathrm{GB} \quad \mathrm{OAV}$}

PC-12 cells were seeded at a density of $1 \times 10^{4}$ cells per well in non-fluorescent collagen coated 96 -well plates one day before the experiments. The cells were incubated in DMEM without L-glutamine and phenol red containing $1.0 \% \mathrm{FBS}, 0.5 \% \mathrm{HS}$ and 13-HPODE (final conc. of $10 \mu \mathrm{M}$ ) and the herb extracts at $37^{\circ} \mathrm{C}$. After $1 \mathrm{hr}$ incubation, the fluorescence of each well was measured with a Titertek Fluorskan II (Flow Laboratories, Rockville, MD, U.S.A.)

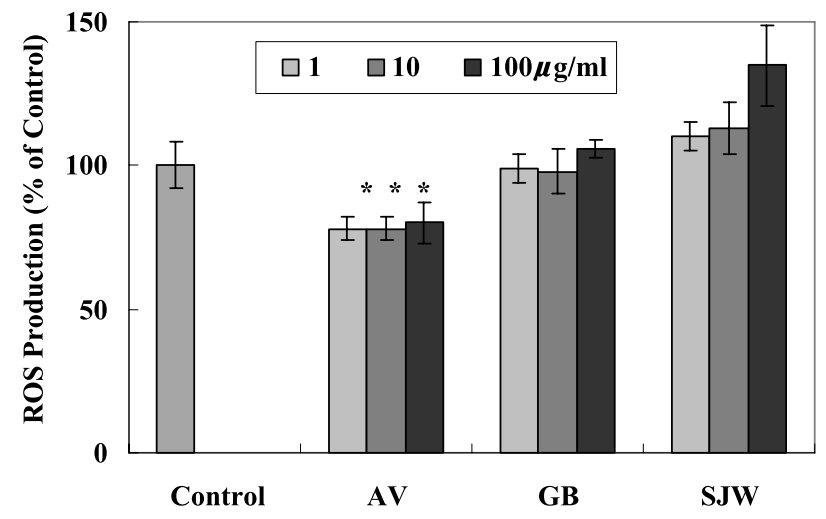

Fig. 2. Effect of pretreatment with herb extract on 13HPODE-induced intracellular production of ROS in PC-12 cells.

$\mathrm{PC} 12$ cells were treated at $37^{\circ} \mathrm{C}$ for $4 \mathrm{~h}$ with herb extract. After the incubation, the cells were washed 2 times with (-) phosphate-buffered saline (PBS) containing 2\% FBS and then incubated in DMEM without L-glutamine and phenol red containing $1.0 \% \mathrm{FBS}, 0.5 \% \mathrm{HS}$ and $50 \mu \mathrm{M}$ DCFH-DA for an additional $45 \mathrm{~min}$ at $37^{\circ} \mathrm{C}$. The cells were washed and maintained in DMEM without L-glutamine and phenol red containing $1.0 \% \mathrm{FBS}$ and $0.5 \% \mathrm{HS}(100 \mu \mathrm{l})$. After adding 13-HPODE $(100 \mu \mathrm{l}$, final conc. of $10 \mu \mathrm{M})$, the fluorescence was monitored after $1 \mathrm{hr}$ incubation. The other procedures were the same as those described in the legend of Fig. 1.

accumulation of quercetin and its metabolites in brain tissue using rats fed quercetin-rich diet. Role of oxidative stress in cellular events of mental stress-related diseases including depression will be clarified in near future. 


\section{ACKNOWLEDGEMENTS}

We are thankful to Dr. S. Seo, Tokiwa Phytochemical Co. Ltd, for his kind gift of the herb extracts. This study was supported by a grant-in aid for the 21 th Century COE program, Human Nutritional Science on Stress Control, The University of Tokushima, Japan.

\section{REFERENCES}

1) Blumenthal $\mathrm{M}:$ The $\mathrm{ABC}$ clinical guide to herbs, American Botanical Council, Austin, Texas, 2003

2) Butterweck V : Antidepressant effects of Apocynum venetum leaves in a forced swimming test. Biol Pharm Bull 24 : 848-851, 2001

3) Blois MS : Antioxidant determinations by the use of a stable free radical. Nature 181 : 11991200, 1958

4) Wang H, Joseph JA:Quantifying cellular oxidative stress by dichlorofluorescein assay using microplate reader. Free Radic Biol Med 27 : 612616, 1999

5) Shirai M, Yamanishi R, Moon J-H, Murota M, Terao J: Effect of quercetin and its conjugated metabolite on the hydrogen peroxide-induced intracellular production of reactive oxygen species in mouse fibroblasts. Biosci Biotechnol Biochem 66 : 1015-1021, 2002

6) Youdim KA, Shukitt-Hale B, Joseph J:Flavonoids and the brain:interactions at blood-brain barrier and their physiological effects on the central nervous system. Free Radic Biol Med 37 : 1683-1693, 2004 